\title{
Meta-analyses of genome-wide association studies identify multiple loci associated with pulmonary function
}

\author{
Dana B Hancock ${ }^{1,29 *}$, Mark Eijgelsheim ${ }^{2,29}$, Jemma B Wilk ${ }^{3,29}$, Sina A Gharib ${ }^{4,5,29}$, Laura R Loehr ${ }^{1,6}$, \\ Kristin D Marciante ${ }^{5,7}$, Nora Franceschini ${ }^{6}$, Yannick M T A van Durme ${ }^{2,8}$, Ting-hsu Chen ${ }^{9,10}$, \\ R Graham Barr ${ }^{11-13}$, Matthew B Schabath ${ }^{14}$, David J Couper ${ }^{15}$, Guy G Brusselle ${ }^{2,8}$, Bruce M Psaty ${ }^{5,7,16-18}$, \\ Cornelia M van Duijn ${ }^{2}$, Jerome I Rotter ${ }^{19}$, André G Uitterlinden ${ }^{2,20}$, Albert Hofman ${ }^{2}$, Naresh M Punjabi ${ }^{21}$, \\ Fernando Rivadeneira $^{2,20}$, Alanna C Morrison ${ }^{22}$, Paul L Enright ${ }^{23}$, Kari E North ${ }^{6,24}$, Susan R Heckbert ${ }^{7,16,17}$, \\ Thomas Lumley ${ }^{25,30}$, Bruno H C Stricker $2,20,26,27,30$, George T O’Connor ${ }^{9,10,30}$ \& Stephanie J London ${ }^{1,28,30}$
}

Spirometric measures of lung function are heritable traits that reflect respiratory health and predict morbidity and mortality. We meta-analyzed genome-wide association studies for two clinically important lung-function measures: forced expiratory volume in the first second $\left(\mathrm{FEV}_{1}\right)$ and its ratio to forced vital capacity $(\mathrm{FEV} / \mathrm{FVC})$, an indicator of airflow obstruction. This meta-analysis included 20,890 participants of European ancestry from four CHARGE Consortium studies: Atherosclerosis Risk in Communities, Cardiovascular Health Study, Framingham Heart Study and Rotterdam Study. We identified eight loci associated with FEV $/$ /FVC

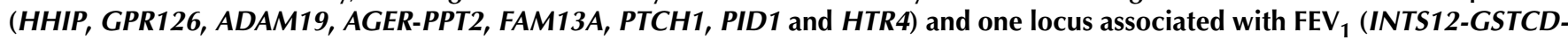
NPNT) at or near genome-wide significance $\left(P<5 \times 10^{-8}\right)$ in the CHARGE Consortium dataset. Our findings may offer insights into pulmonary function and pathogenesis of chronic lung disease.

Pulmonary function is an easily measurable and reliable index of the physiological state of the lungs and airways ${ }^{1}$. Pulmonary function also predicts mortality in the general population, even among people who have never smoked (never-smokers) who have only modestly reduced pulmonary function and no respiratory symptoms ${ }^{2,3}$. The peak level of pulmonary function attained in early adulthood and its subsequent decline with age are likely influenced by genetic and environmental factors. Tobacco smoking is a major environmental cause of accelerated decline in pulmonary function with age. Other inhaled pollutants also appear to contribute. Familial aggregation studies suggest a genetic contribution to lung function, with heritability estimates exceeding $40 \%{ }^{4,5}$, but little is known about the specific genetic factors involved. A relatively uncommon deficiency of $\alpha 1$-antitrypsin is the only established genetic risk factor for accelerated decline in pulmonary function and for development of chronic obstructive pulmonary disease (COPD), especially in smokers ${ }^{4,6}$. However, $\alpha 1$-antitrypsin accounts for little of the population variability in pulmonary function ${ }^{4}$. Candidate gene studies suggest that other genetic variants may influence the time course of pulmonary function and its decline in relation to smoking, but these putative genetic risk factors remain unknown ${ }^{4}$.

Forced expiratory volume in the first second $\left(\mathrm{FEV}_{1}\right)$ and its ratio to forced vital capacity $\left(\mathrm{FEV}_{1} / \mathrm{FVC}\right)$ are two clinically relevant pulmonary function measures. Although both $\mathrm{FEV}_{1}$ and $\mathrm{FVC}$ are influenced by lung size and can be reduced by restrictive lung diseases, obstructive lung disease leads to proportionately greater reduction in $\mathrm{FEV}_{1}$ than FVC. Therefore, reduced $\mathrm{FEV}_{1} / \mathrm{FVC}$, an indicator of airflow obstruction that is independent of lung size, is the primary criterion for defining an obstructive ventilatory defect ${ }^{1}$. Whereas low $\mathrm{FEV}_{1} / \mathrm{FVC}$ indicates the presence of airflow obstruction, $\mathrm{FEV}_{1}$ is used to classify the severity and follow the progression of obstructive lung disease over time $e^{5,7,8}$.

The first genome-wide association study (GWAS) for pulmonary function evaluating 70,987 SNPs in about 1,220 Framingham Heart Study (FHS) participants revealed no genome-wide significant loci ${ }^{9}$. Recently, a GWAS of $\mathrm{FEV}_{1}$ /FVC using 2,540,223 SNPs in 7,691 FHS participants identified several SNPs on chromosome $4 \mathrm{q} 31$ near HHIP with genome-wide significance ${ }^{10}$. A GWAS of COPD ${ }^{11}$ also implicated the HHIP region along with CHRNA3-CHRNA5 on chromosome 15, a region previously associated with nicotine dependence ${ }^{12,13}$.

We conducted meta-analyses of GWAS results for a cross-sectional analysis of pulmonary function $\left(\mathrm{FEV}_{1} / \mathrm{FVC}\right.$ and $\left.\mathrm{FEV}_{1}\right)$ in 20,890 individuals of European ancestry from four Cohorts for Heart and Aging Research in Genomic Epidemiology (CHARGE) Consortium ${ }^{14}$ studies: Atherosclerosis Risk in Communities (ARIC), Cardiovascular Health Study (CHS), FHS and Rotterdam Study (RS-I and RS-II). Given that cigarette smoking is a major risk factor for pulmonary function decline, we conducted meta-analyses with adjustment for smoking status and quantity (pack-years), and in subgroups of those who have ever smoked (ever-smokers) and never-smokers. Loci meeting genomewide significance and other selected high-signal hits were evaluated for replication with the SpiroMeta consortium, an independent consortium

*A full list of authors' affiliations appears at the end of the paper. 
Table 1 Characteristics of cohort participants in the CHARGE consortium at the time of pulmonary function assessment

\begin{tabular}{|c|c|c|c|c|c|c|c|c|c|c|c|c|c|c|c|c|c|}
\hline \multirow{3}{*}{$\begin{array}{l}\text { Cohort, } \\
\text { number }\end{array}$} & \multirow[b]{3}{*}{$\%$ male } & \multirow{2}{*}{\multicolumn{2}{|c|}{ Age (y) }} & \multirow{2}{*}{\multicolumn{2}{|c|}{ Height (m) }} & \multirow{2}{*}{\multicolumn{2}{|c|}{$\mathrm{BMI}\left(\mathrm{kg} / \mathrm{m}^{2}\right)$}} & \multicolumn{3}{|c|}{ Current smoking } & \multicolumn{3}{|c|}{ Former smoking } & \multicolumn{4}{|c|}{ Pulmonary function } \\
\hline & & & & & & & & \multirow[b]{2}{*}{$\%$} & \multicolumn{2}{|c|}{ Pack-years } & \multirow[b]{2}{*}{$\%$} & \multicolumn{2}{|c|}{ Pack-years } & \multicolumn{2}{|c|}{$\mathrm{FEV}_{1}(\mathrm{ml})$} & \multicolumn{2}{|c|}{$\mathrm{FEV}_{1} / \mathrm{FVC}(\%)$} \\
\hline & & Mean & s.d. & Mean & s.d. & Mean & s.d. & & Mean & s.d. & & Mean & s.d. & Mean & s.d. & Mean & s.d. \\
\hline \multicolumn{18}{|l|}{7,980} \\
\hline $\mathrm{CHS}$ & 39.0 & 72.3 & 5.4 & 1.65 & 0.09 & 26.3 & 4.4 & 10.8 & 45.1 & 25.4 & 40.0 & 29.9 & 26.4 & 2,116 & 659 & 70.5 & 10.5 \\
\hline \multicolumn{18}{|l|}{3,140} \\
\hline RS-I & 45.4 & 74.5 & 5.6 & 1.67 & 0.09 & 27.4 & 4.0 & 11.7 & 40.1 & 23.4 & 58.8 & 24.7 & 22.5 & 2,320 & 728 & 73.1 & 8.3 \\
\hline \multicolumn{18}{|l|}{1,224} \\
\hline RS-II & 44.7 & 67.2 & 6.3 & 1.68 & 0.09 & 27.7 & 4.1 & 14.0 & 37.4 & 21.4 & 52.3 & 21.5 & 22.0 & 2,716 & 782 & 75.8 & 9.1 \\
\hline
\end{tabular}

Genotyping platforms used: ARIC, Affymetrix GeneChip SNP Array 6.0; CHS, Illumina Human 370CNV BeadChip; FHS, Affymetrix GeneChip Human Mapping 500K Array and 50K Human Gene Focused Panel; RS-I, Illumina Infinium II HumanHap 550K Single and Duo Bead Chips; RS-II, IIlumina Infinium II HumanHap 500K Dup and $610 K$ Quad Bead Chips. $\mathrm{FEV}_{1}$, forced expiratory volume in one second; $F E V_{1} / F V C$, ratio of forced expiratory volume in one second to forced vital capacity.

having a combined GWAS sample size of 20,288 participants of European ancestry as described in the companion paper ${ }^{15}$.

\section{RESULTS}

Meta-analyses of CHARGE genome-wide association results

Meta-analyses for $\mathrm{FEV}_{1} / \mathrm{FVC}$ and $\mathrm{FEV}_{1}$ were conducted using approximately 2,534,500 SNPs in 20,890 CHARGE participants of European ancestry ( $n=7,980$ from ARIC, $n=3,140$ from CHS, $n=7,694$ from FHS, $n=1,224$ from RS-I and $n=852$ from RS-II) and in subgroups of ever- $(n=11,963)$ and never-smokers $(n=8,927)$. Characteristics of the cohort participants are presented in Table 1. We applied genomic control, although cohort-specific genomic inflation factors $\left(\lambda_{\mathrm{gc}}\right)$ were low (for $\mathrm{FEV}_{1} / \mathrm{FVC}$ ranging from 1.00 (RS-I and RS-II) to 1.05 (ARIC) and for FEV $_{1}$ ranging from 1.01 (RS-II) to 1.05 (FHS)), suggesting minimal population stratification. The metaanalysis $\lambda_{\mathrm{gc}}$ was 1.04 for $\mathrm{FEV}_{1} / \mathrm{FVC}$ and 1.03 for $\mathrm{FEV}_{1}$ in all participants. Quantile-quantile (Q-Q) plots show large deviations between observed and expected $P$ values for high-signal SNPs in analyses of $\mathrm{FEV}_{1} / \mathrm{FVC}$ and $\mathrm{FEV}_{1}$ in all participants (Supplementary Fig. 1a,b), $\mathrm{FEV}_{1} / \mathrm{FVC}$ in never-smokers (Supplementary Fig. 2a), and $\mathrm{FEV}_{1}$ in ever-smokers (Supplementary Fig. 3c). Genome-wide significant associations $\left(P<5 \times 10^{-8}\right)$ were found for multiple SNPs in each of these analyses (see Fig. 1a,b for overall analyses and Supplementary Fig. 2b,d and Supplementary Fig. 3b,d for analyses stratified by ever or never smoking). The top 2,000 SNPs associated with each measure, $\mathrm{FEV}_{1} / \mathrm{FVC}$ and $\mathrm{FEV}_{1}$, beyond genome-wide significance $\left(P>5 \times 10^{-8}\right)$ are presented in Supplementary Table 1.

For $\mathrm{FEV}_{1} / \mathrm{FVC}$, genome-wide significant associations were seen for 119 SNPs at seven loci (Supplementary Table 2). The SNP with the smallest $P$ value, rs $1980057\left(P=4.90 \times 10^{-11}\right)$, is located on chromosome $4 \mathrm{q} 31.2281 \mathrm{~kb}$ away from the $5^{\prime}$ end of HHIP. There were 27 other genome-wide significant SNPs in the HHIP region (Fig. 2a). Additionally, 69 genome-wide significant SNPs were located in or near the $3^{\prime}$ end of GPR126 on chromosome 6q24.1, with the top SNP (rs3817928) having $P=2.60 \times 10^{-10}$ (Fig. 2b). Fifty-nine of these 69 GPR126 SNPs were associated with $\mathrm{FEV}_{1} / \mathrm{FVC}$ at genome-wide significance among never-smokers (Supplementary Table 2). Seven SNPs on chromosome 5q33.3 located in ADAM19 (Fig. 2c), two correlated SNPs on chromosome 6p21.32 $\left(r^{2}=0.66\right.$, Fig. 2 d $)$ located in two genes (AGER and PPT2), four SNPs on chromosome 4q22.1 near the 5' end of FAM13A (Fig. 2e), two SNPs on chromosome 9q22.32 in PTCH1 (Fig. 2f), and six SNPs on chromosome 2q36.3 near the $3^{\prime}$ end of PID1 (Fig. 2g) were also significantly associated with $\mathrm{FEV}_{1} / \mathrm{FVC}$ in all participants. SNPs in AGER, PPT2, PTCH1 and PID1 had minor allele frequencies (MAFs) between $4 \%$ and $10 \%$, whereas all other significantly associated SNPs had MAFs exceeding 10\%. Absolute values of $\beta$ (per-allele change) for $\mathrm{FEV}_{1} / \mathrm{FVC}$ ranged from $0.44 \%$ to $1.14 \%$. The directions of $\beta$ were consistent across the CHARGE cohorts for all genome-wide significant SNPs except for the GPR126 SNPs noted in Supplementary Table 2. A borderline significant association $\left(P=5.37 \times 10^{-8}, \mathrm{MAF}=0.42, \beta=-0.43\right)$ with $\mathrm{FEV}_{1} / \mathrm{FVC}$ was noted for the chromosome 5q33.1 SNP rs11168048 in HTR4 (Fig. 2h).
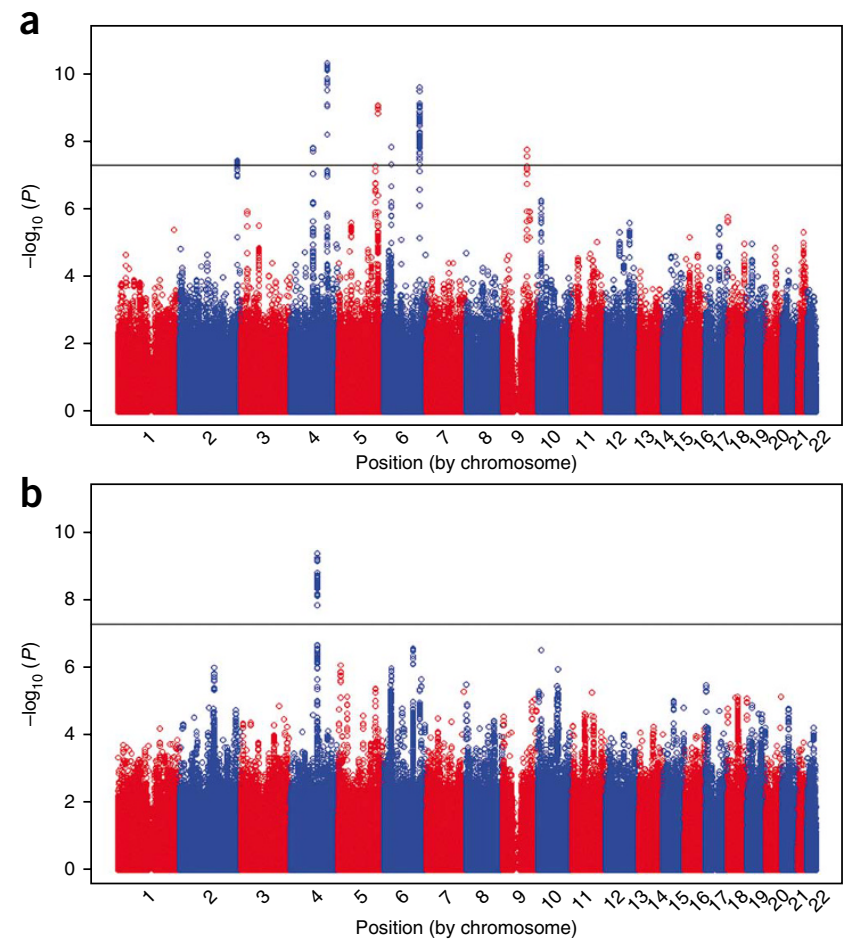

Figure 1 Meta-analyses of approximately 2,534,500 SNPs tested for association with pulmonary function measures in all participants from the

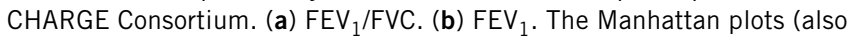
known as $-\log _{10}(P)$ association plots) show the chromosomal position of SNPs exceeding the genome-wide significance threshold $\left(P<5 \times 10^{-8}\right.$ as indicated by the solid black line). 

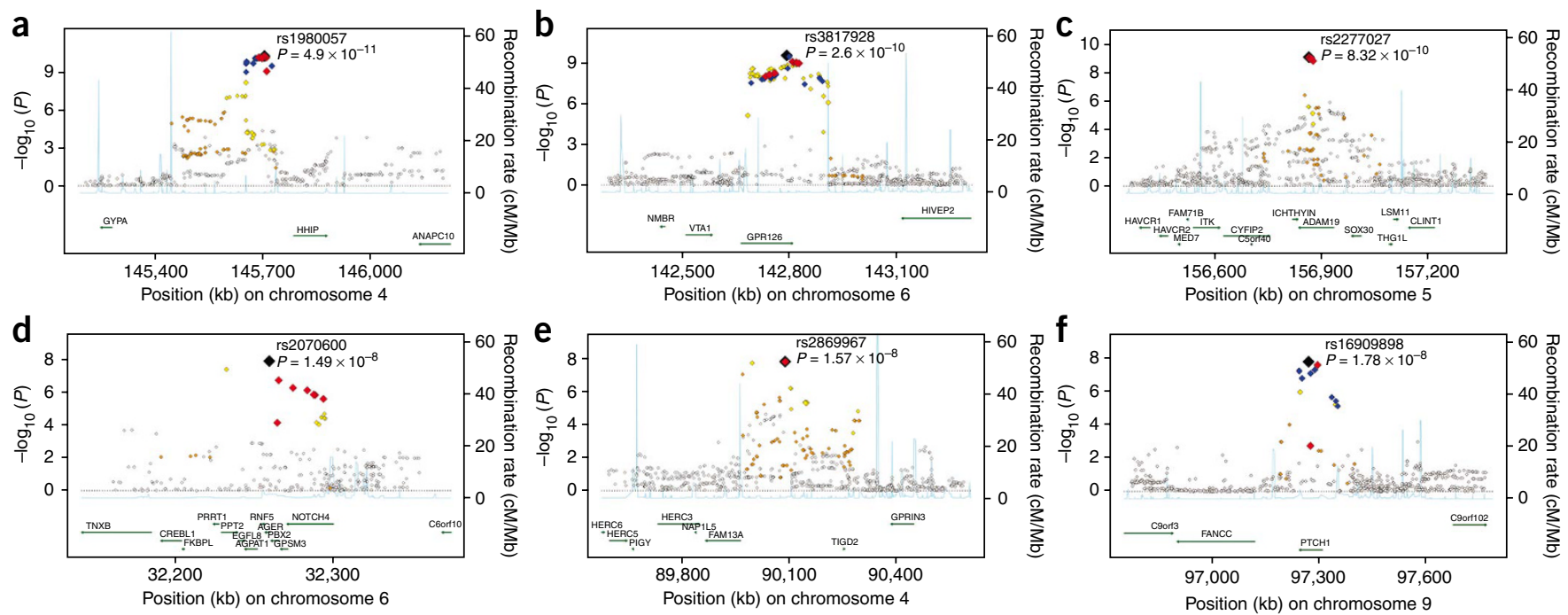

Figure 2 Regional association plots for loci associated with $\mathrm{FEV}_{1} / \mathrm{FVC}$ in the CHARGE consortium at or near genome-wide significance. (a-h) Loci included HHIP on chromosome 4q31.22 (a), GPR126 on chromosome 6q24.1 (b), ADAM19 on chromosome 5q33.3 (c), AGER-PPT2 on chromosome 6p21.32 (d), FAM13A on chromosome 4q22.1 (e), PTCH1 on chromosome 9q22.32 (f), PID1 on chromosome
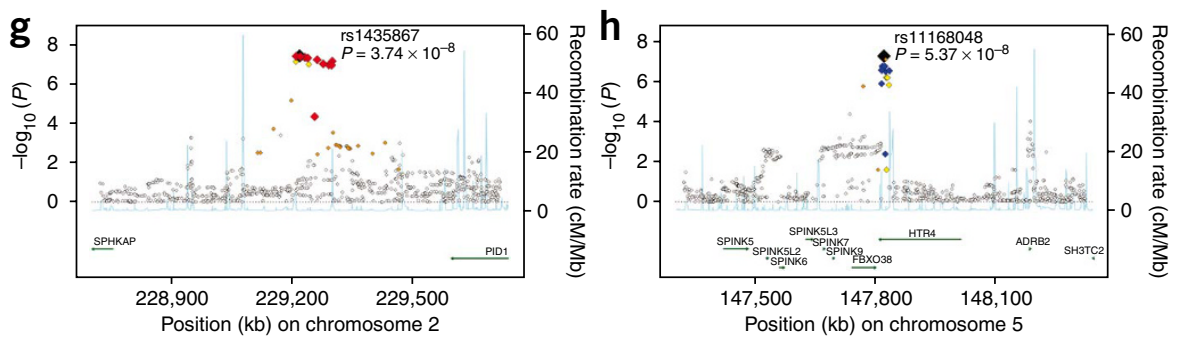

2q36.3 (g) and HTR4 on chromosome 5q33.1 (h). For each locus, correlations between the target SNP (the SNP with the lowest $P$ value, depicted in black) and other SNPs in the region are depicted in red when $r^{2}=1$, blue when $0.8 \leq r^{2}<1$, yellow when $0.5 \leq r^{2}<0.8$, orange when $0.2 \leq r^{2}<0.5$ and white when $r^{2}<0.2$. The $r^{2}$ values were based on the HapMap CEU population. Gene annotations are shown in green, and estimated recombination rates from HapMap are shown in light blue.

Cohort-specific association results for SNPs with the smallest $P$ value from each locus implicated at or near genome-wide significance are shown in Supplementary Table 3.

For $\mathrm{FEV}_{1}$, genome-wide significant associations were observed for 46 SNPs on chromosome $4 \mathrm{q} 24$ in or near four adjacent genes (Supplementary Table 4 ). The SNP with the smallest $P$ value, rs17331332 $\left(P=4.00 \times 10^{-10}\right)$, is located near NPNT. The 45 other

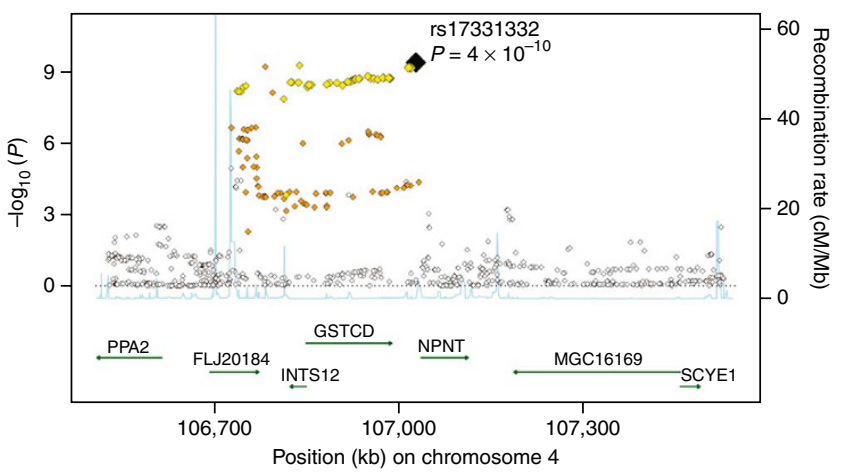

Figure 3 Regional association plot for the chromosome 4q24 locus associated with $\mathrm{FEV}_{1}$ in the CHARGE consortium at genome-wide significance, which includes FLJ20184, INTS12, GSTCD and NPNT. Correlations between the target SNP (the SNP with the lowest $P$ value, depicted in black) and other SNPs in the region are depicted in red when $r^{2}=1$, blue when $0.8 \leq r^{2}<1$, yellow when $0.5 \leq r^{2}<0.8$, orange when $0.2 \leq r^{2}<0.5$ and white when $r^{2}<0.2$. The $r^{2}$ values were based on the HapMap CEU population. Gene annotations are shown in green, and estimated recombination rates from HapMap are shown in light blue. significantly associated SNPs include four SNPs located near the $5^{\prime}$ end of NPNT, 5 SNPs located in INTS12 or near its $3^{\prime}$ end, seven SNPs located in FLJ20184 or near its $3^{\prime}$ end and 29 SNPs located in GSTCD. FLJ20184 encodes a hypothetical protein, according to several genome browsers including the UCSC Genome Browser (see URLs) ${ }^{16}$, but there is no approved Human Gene Organization (HUGO) gene name for this locus ${ }^{17}$. The SNP rs17331332 is correlated at $r^{2}>0.5$ with most other significantly associated SNPs in this region (Fig. 3), suggesting that the associations in the four adjacent genes represent one independent finding. The significantly associated SNPs had MAFs between $6 \%$ and $8 \%$. The absolute $\beta$ (per-allele change) values for $\mathrm{FEV}_{1}$ ranged from 55.92 to $71.43 \mathrm{ml}$ (Supplementary Table 4), and the $\beta$ directions were consistent across the CHARGE cohorts for all 46 genome-wide significant SNPs (Supplementary Table 3 for rs17331332). Among these 46 SNPs, 39 were associated with $\mathrm{FEV}_{1}$ at genome-wide significance among ever-smokers (Supplementary Table 4).

To evaluate whether other loci may also influence pulmonary function, we created Q-Q plots for $\mathrm{FEV}_{1} / \mathrm{FVC}$ and $\mathrm{FEV}_{1}$ among all participants after removing SNPs at or close to genome-wide significance and nearby SNPs correlated at $r^{2}>0.2$ with the top SNP for each locus (totaling 1,862 SNPs removed for $\mathrm{FEV}_{1} / \mathrm{FVC}$ and $284 \mathrm{SNPs}$ removed for $\mathrm{FEV}_{1}$ ). The resulting Q-Q plots show some excess of small $P$ values for $\mathrm{FEV}_{1} / \mathrm{FVC}$ (Supplementary Fig. 4a) and $\mathrm{FEV}_{1}$ (Supplementary Fig. 4b) over expectation.

\section{Putative functional polymorphisms}

Three SNPs among the 119 genome-wide significant SNPs for $\mathrm{FEV}_{1}$ / FVC are nonsynonymous (missense) polymorphisms: rs11155242 
Table 2 Joint meta-analysis of SNPs selected from the top 8 loci implicated for FEV 1 /FVC in the CHARGE GWAS and tested for replication with $\mathrm{FEV}_{1} / \mathrm{FVC}$ in the SpiroMeta consortium ${ }^{15}$

\begin{tabular}{|c|c|c|c|c|c|c|c|c|c|c|c|}
\hline \multirow[b]{2}{*}{ SNP } & \multirow[b]{2}{*}{ Chr. } & \multirow[b]{2}{*}{ Gene } & \multirow{2}{*}{$\begin{array}{l}\text { Ref. } \\
\text { allele }\end{array}$} & \multicolumn{3}{|c|}{ CHARGE GWAS } & \multicolumn{3}{|c|}{ SpiroMeta replication } & \multicolumn{2}{|c|}{ Joint meta-analysis } \\
\hline & & & & Allele freq. ${ }^{a}$ & $\beta(\%)$ & $P$ & Allele freq. ${ }^{a}$ & $\beta(\%)$ & $P$ & $\beta(\%)$ & $P$ \\
\hline rs1980057 & 4 & HHIP & $\mathrm{T}$ & 0.40 & 0.51 & $4.90 \times 10^{-11}$ & 0.45 & 0.54 & $1.09 \times 10^{-10}$ & 0.52 & $3.21 \times 10^{-20}$ \\
\hline rs1032295 & 4 & HHIP & $\mathrm{T}$ & 0.58 & -0.47 & $6.28 \times 10^{-9}$ & 0.55 & -0.46 & $1.15 \times 10^{-7}$ & -0.47 & $4.37 \times 10^{-15}$ \\
\hline rs3817928 & 6 & GPR126 & $A$ & 0.78 & -0.59 & $2.60 \times 10^{-10}$ & 0.79 & -0.21 & $3.99 \times 10^{-2}$ & -0.42 & $1.17 \times 10^{-9}$ \\
\hline rs7776375 & 6 & GPR126 & $A$ & 0.71 & -0.51 & $1.33 \times 10^{-9}$ & 0.73 & -0.19 & $4.87 \times 10^{-2}$ & -0.37 & $6.71 \times 10^{-9}$ \\
\hline rs6937121 & 6 & GPR126 & $\mathrm{T}$ & 0.71 & -0.49 & $2.46 \times 10^{-9}$ & 0.72 & -0.18 & $5.62 \times 10^{-2}$ & -0.35 & $1.25 \times 10^{-8}$ \\
\hline rs11155242 & 6 & GPR126 & $A$ & 0.80 & -0.54 & $9.13 \times 10^{-9}$ & 0.80 & -0.16 & $1.26 \times 10^{-1}$ & -0.37 & $1.45 \times 10^{-7}$ \\
\hline rs2277027 & 5 & $A D A M 19$ & $A$ & 0.71 & 0.49 & $8.32 \times 10^{-10}$ & 0.66 & 0.24 & $5.04 \times 10^{-3}$ & 0.38 & $9.93 \times 10^{-11}$ \\
\hline rs1422795 & 5 & $A D A M 19$ & $\mathrm{~T}$ & 0.66 & 0.48 & $1.16 \times 10^{-9}$ & 0.66 & 0.24 & $6.65 \times 10^{-3}$ & 0.37 & $2.62 \times 10^{-10}$ \\
\hline rs2070600 & 6 & $A G E R^{\mathrm{b}}$ & $\mathrm{T}$ & 0.04 & 1.06 & $1.49 \times 10^{-8}$ & 0.06 & 0.94 & $4.40 \times 10^{-7}$ & 1.00 & $3.15 \times 10^{-14}$ \\
\hline rs10947233 & 6 & PPT2 ${ }^{\mathrm{b}}$ & $\mathrm{T}$ & 0.04 & 1.14 & $4.71 \times 10^{-8}$ & 0.04 & 1.05 & $3.43 \times 10^{-5}$ & 1.10 & $6.66 \times 10^{-12}$ \\
\hline rs2869967 & 4 & $F A M 13 A$ & $\mathrm{~T}$ & 0.61 & 0.44 & $1.57 \times 10^{-8}$ & 0.59 & 0.13 & $1.28 \times 10^{-1}$ & 0.30 & $1.91 \times 10^{-7}$ \\
\hline rs6830970 & 4 & FAM13A & $A$ & 0.65 & 0.46 & $1.92 \times 10^{-8}$ & 0.64 & 0.11 & $2.00 \times 10^{-1}$ & 0.30 & $6.63 \times 10^{-7}$ \\
\hline rs16909898 & 9 & PTCH1 & $A$ & 0.90 & 0.77 & $1.78 \times 10^{-8}$ & 0.89 & 0.20 & $1.70 \times 10^{-1}$ & 0.50 & $5.34 \times 10^{-7}$ \\
\hline rs10512249 & 9 & PTCH1 & $A$ & 0.10 & -0.73 & $2.79 \times 10^{-8}$ & 0.10 & -0.19 & $1.67 \times 10^{-1}$ & -0.48 & $5.75 \times 10^{-7}$ \\
\hline rs1435867 & 2 & PID1 & $\mathrm{T}$ & 0.93 & -0.84 & $3.74 \times 10^{-8}$ & 0.93 & -0.075 & $6.51 \times 10^{-1}$ & -0.50 & $1.53 \times 10^{-5}$ \\
\hline rs10498230 & 2 & PID1 & $\mathrm{T}$ & 0.07 & 0.86 & $3.87 \times 10^{-8}$ & 0.07 & 0.075 & $6.56 \times 10^{-1}$ & 0.50 & $1.46 \times 10^{-5}$ \\
\hline rs11168048 & 5 & HTR4 & $\mathrm{T}$ & 0.58 & -0.43 & $5.37 \times 10^{-8}$ & 0.58 & -0.36 & $3.97 \times 10^{-5}$ & -0.40 & $1.08 \times 10^{-11}$ \\
\hline rs7735184 & 5 & HTR4 & $\mathrm{T}$ & 0.40 & 0.40 & $1.74 \times 10^{-7}$ & 0.41 & 0.34 & $5.62 \times 10^{-5}$ & 0.37 & $6.23 \times 10^{-11}$ \\
\hline
\end{tabular}

'Gene' indicates gene either harboring the SNP or nearest to it. SNPS are grouped together by the nearest gene and ordered by the CHARGE GWAS $P$ value. $P$ values highlighted in bold exceeded the threshold for significance $\left(P<5 \times 10^{-8}\right.$ for the GWAS and joint meta-analysis, $P<8.33 \times 10^{-4}$ for replication). $\beta$, per-allele change in FEV ${ }_{1} / \mathrm{FVC}$. Chr., chromosome. aWeighted average reference allele frequency for combined cohorts. ${ }^{\mathrm{b}} \mathrm{AGER}$ and $P$ PT2 SNPS are considered to represent one locus given their correlations. FEV ${ }_{1} / \mathrm{FVC}$, forced expiratory volume in $1 \mathrm{~s}$ to forced vital capacity ratio.

(resulting in a lysine-to-glutamine substitution) in GPR126, rs1422795 (serine-to-glycine substitution) in ADAM19 and rs2070600 (glycineto-serine substitution) in AGER. The Polymorphism Phenotyping (PolyPhen) program (see URLs) ${ }^{18}$ predicts that the amino acid substitutions resulting from rs11155242 and rs1422795 cause benign changes but that rs2070600 has a possibly damaging impact on the structure and function of AGER.

All other $\mathrm{SNPs}$ implicated for $\mathrm{FEV}_{1} / \mathrm{FVC}$ or $\mathrm{FEV}_{1}$ are intergenic, intronic or located in $3^{\prime}$ untranslated regions. Of these, three intronic GPR126SNPs (rs9496346, rs1040525 and rs6929442) and one intergenic SNP near NPNT (rs10516529) are located in transcription factor binding sites, according to the UCSC Genome Browser (see URLs) ${ }^{16}$.

\section{Replication with the SpiroMeta consortium}

Thirty high-signal SNPs associated with $\mathrm{FEV}_{1} / \mathrm{FVC}$ (18 SNPs from eight loci) or $\mathrm{FEV}_{1}$ (12 SNPs from three loci) at or close to genomewide significance were tested for replication in the SpiroMeta consortium, with results reported in a companion paper ${ }^{15}$. We evaluated these SNPs in 16,178 SpiroMeta participants of European ancestry with complete quantitative smoking data using the CHARGE analytic method (see statistical analysis section of Online Methods), which included adjustment for smoking status and pack-years, and performed joint meta-analyses of CHARGE GWAS and SpiroMeta replication results (Tables 2 and 3). $P$ values that exceeded the significance threshold in SpiroMeta $\left(P<8.33 \times 10^{-4}\right.$ based on 60 tests $)$

Table 3 Joint meta-analysis of SNPs selected from the top three loci implicated for FEV in $_{1}$ the CHARGE GWAS and tested for replication with $\mathrm{FEV}_{1}$ in the SpiroMeta consortium ${ }^{15}$

\begin{tabular}{|c|c|c|c|c|c|c|c|c|c|c|c|}
\hline \multirow[b]{2}{*}{$\underline{\text { SNP }}$} & \multirow[b]{2}{*}{ Chr. } & \multirow[b]{2}{*}{ Gene } & \multirow{2}{*}{$\begin{array}{l}\text { Ref. } \\
\text { allele }\end{array}$} & \multicolumn{3}{|c|}{ CHARGE GWAS } & \multicolumn{3}{|c|}{ SpiroMeta replication } & \multicolumn{2}{|c|}{ Joint meta-analysis } \\
\hline & & & & Allele freq. ${ }^{a}$ & $\beta$ & $P$ & Allele freq. ${ }^{a}$ & $\beta$ & $P$ & $\beta$ & $P$ \\
\hline rs17331332 & 4 & $N P N T^{D}$ & A & 0.08 & 60.35 & $4.00 \times 10^{-10}$ & 0.07 & 52.11 & $2.45 \times 10^{-6}$ & 56.79 & $5.69 \times 10^{-15}$ \\
\hline rs17036341 & 4 & $N P N T^{D}$ & C & 0.93 & -58.85 & $6.29 \times 10^{-10}$ & 0.94 & -53.82 & $6.38 \times 10^{-7}$ & -56.65 & $2.18 \times 10^{-15}$ \\
\hline rs17036090 & 4 & INTS12 $2^{\mathrm{b}}$ & $\mathrm{T}$ & 0.93 & -56.6 & $1.37 \times 10^{-8}$ & 0.94 & -59.51 & $7.85 \times 10^{-8}$ & -57.90 & $5.61 \times 10^{-15}$ \\
\hline rs17036052 & 4 & FLJ20184b & $\mathrm{T}$ & 0.06 & 71.43 & $6.07 \times 10^{-10}$ & 0.04 & 68.19 & $5.78 \times 10^{-7}$ & 70.08 & $1.83 \times 10^{-15}$ \\
\hline rs11728716 & 4 & $G S T C D^{b}$ & A & 0.07 & 57.52 & $1.80 \times 10^{-9}$ & 0.06 & 57.41 & $7.61 \times 10^{-8}$ & 57.47 & $7.20 \times 10^{-16}$ \\
\hline rs3749893 & 6 & $T S P Y L 4^{\mathrm{c}, \mathrm{d}}$ & A & 0.37 & -26.55 & $5.35 \times 10^{-7}$ & 0.37 & 4.01 & $4.62 \times 10^{-1}$ & -11.71 & $2.05 \times 10^{-3}$ \\
\hline rs 1052443 & 6 & $N T 5 D C 1^{\mathrm{c}, \mathrm{d}}$ & A & 0.62 & 26.12 & $7.50 \times 10^{-7}$ & 0.62 & -3.75 & $4.90 \times 10^{-1}$ & 11.61 & $2.17 \times 10^{-3}$ \\
\hline rs6555465 & 5 & $A D C Y 2^{\mathrm{d}}$ & A & 0.18 & -31.23 & $1.32 \times 10^{-6}$ & 0.19 & 0.12 & $9.86 \times 10^{-1}$ & -16.24 & $4.96 \times 10^{-4}$ \\
\hline rs7710510 & 5 & $A D C Y 2^{\mathrm{d}}$ & $\mathrm{T}$ & 0.19 & -30.34 & $1.72 \times 10^{-6}$ & 0.21 & -2.77 & $6.77 \times 10^{-1}$ & -17.20 & $1.77 \times 10^{-4}$ \\
\hline
\end{tabular}

'Gene' indicates gene either harboring the SNP or nearest to it. SNPs are grouped together by the nearest gene and ordered by the CHARGE GWAS $P$ value. $P$ values highlighted in bold exceeded the threshold for significance $\left(P<5 \times 10^{-8}\right.$ for the GWAS and joint meta-analysis, $P<8.33 \times 10^{-4}$ for replication). $\beta$, per-allele change in FEV $\left(\mathrm{mL}\right.$ ). FEV $\mathrm{F}_{1}$, forced expiratory volume in one second.

aWeighted average reference allele frequency for combined cohorts. ${ }^{b} N P N T$, INTS12, FLJ20184, and GSTCD SNPs are considered to represent one locus given their correlations. ${ }^{c}$ TSPYL4 and NT5DC1 SNPs are considered to represent one locus given their correlations. ${ }^{\mathrm{d}} T S P Y L 4, N T 5 D C 1$ and $A D C Y 2 \mathrm{SNPs}$ were not associated with FEV ${ }_{1}$ at genome-wide significance, but these loci had the next-smallest $P$ values for association. 
or the genome-wide significance threshold in joint meta-analyses $\left(P<5 \times 10^{-8}\right)$ were considered significant evidence for replication at their corresponding SNPs.

For $\mathrm{FEV}_{1} / \mathrm{FVC}$, among 18 SNPs tested for replication, six SNPs in three loci were significantly associated with this measure in SpiroMeta: rs1980057 and rs1032295 near HHIP $\left(r^{2}=0.72\right)$, rs2070600 in AGER and rs10947233 in PPT2 $\left(r^{2}=0.66\right)$, and $\mathrm{rs} 11168048$ and rs7735184 in HTR4 $\left(r^{2}=0.93\right)$ (Table 2$)$. Their joint meta-analysis $P$ values ranged from $3.21 \times 10^{-20}$ to $6.23 \times 10^{-11}$ (Table 2). Five additional SNPs in GPR126 (rs3817928, rs7776375 and rs6937121) and ADAM19 (rs2277027 and rs1422795) were not significantly associated with $\mathrm{FEV}_{1} / \mathrm{FVC}$ at genome-wide significance in SpiroMeta alone, but these SNPs were associated at genome-wide significance in the joint metaanalysis, with $P$ values ranging from $9.93 \times 10^{-11}$ to $1.25 \times 10^{-8}$ (Table 2). For replicated SNPs, the allele frequencies and the direction and magnitude of the associations with $\mathrm{FEV}_{1}$ /FVC were similar between consortia (Table 2). Further, the HHIP, ADAM19 and HTR4 SNPs were significantly associated with $\mathrm{FEV}_{1}$ in SpiroMeta (Supplementary Table 5). The HHIP SNP rs 1980057 and the HTR4 SNPs rs 11168048 and rs7735184 were also associated with $\mathrm{FEV}_{1}$ at genome-wide significance in the joint meta-analysis $\left(P\right.$ ranging from $5.86 \times 10^{-9}$ to $1.58 \times 10^{-8}$, Supplementary Table 5). SNPs in FAM13A, PTCH1 and PID1 that gave genome-wide significance in CHARGE were not confirmed in analyses with SpiroMeta.

For $\mathrm{FEV}_{1}$, among the 12 SNPs tested for replication, eight SNPs from one locus with four adjacent genes were significantly associated with this measure in SpiroMeta, including rs17331332 and rs17036341 near NPNT, rs11727189 and rs17036090 in or near INTS12, rs17036052 and rs17035960 in or near FLJ20184, and rs11097901 and rs11728716 in GSTCD (Table 3). For replicated SNPs, the allele frequencies and the direction and magnitude of the associations with $\mathrm{FEV}_{1}$ were similar between consortia, and $P$ values from joint meta-analysis ranged from $4.66 \times 10^{-17}$ to $9.42 \times 10^{-14}$ (Table 2). None of these SNPs were significantly associated with $\mathrm{FEV}_{1} / \mathrm{FVC}$ in CHARGE or SpiroMeta (Supplementary Table 5).

\section{Associations in individuals with normal pulmonary function}

To address whether the genetic associations hold even among people with normal pulmonary function, we repeated the meta-analyses after excluding individuals with asthma or COPD, leaving 17,855 individuals ( $n=6,912$ from ARIC, $n=2,634$ from CHS, $n=6,371$ from FHS, $n=1,126$ from RS-I and $n=812$ from RS-II). Asthma was defined by self-report of ever having asthma or self-report of ever having physician-diagnosed asthma. COPD was defined spirometrically as having both $\mathrm{FEV}_{1} / \mathrm{FVC}$ and $\mathrm{FEV}_{1}$ less than the lower limit of normal values using National Health and Nutrition Examination Survey III prediction equations ${ }^{19,20}$. Comparing the original meta-analyses to the meta-analyses with exclusions for asthma and COPD, $\beta$ estimates were highly correlated for the high-signal SNPs tested for replication (Pearson's $r>0.99$ for $18 \mathrm{FEV}_{1} / \mathrm{FVC}$ SNPs and $12 \mathrm{FEV}_{1} \mathrm{SNPs}_{\text {). }}$ $\beta$ estimates remained highly correlated for SNPs with $P$ values as high as 0.01 in the original meta-analyses $\left(r=0.92\right.$ for $\mathrm{FEV}_{1} / \mathrm{FVC}$ and $r=0.96$ for $\mathrm{FEV}_{1}$ ). As expected, there was some attenuation in $P$ values for many of the SNPs in our implicated loci given the substantial power loss due to both reduced sample size and the truncation of the $\mathrm{FEV}_{1} / \mathrm{FVC}$ and $\mathrm{FEV}_{1}$ distributions, but there was substantial overlap in the top-ranking SNPs between the two meta-analyses (data not shown). The $P$ values for some topranking SNPs became smaller, including several ADAM19, FAM13A and HTR4 SNPs associated with $\mathrm{FEV}_{1} / \mathrm{FVC}$. Of note, 12 SNPs in HTR4, a locus with one SNP, rs11168048, showing borderline genome-wide significance in the original meta-analysis showed genome-wide significance in the subset of individuals without asthma or COPD $\left(P=6.93 \times 10^{-9}\right.$ for rs11168048).

\section{DISCUSSION}

In meta-analyses of GWAS results in 20,890 CHARGE participants of European ancestry, we identified genome-wide significant associations with $\mathrm{FEV}_{1} / \mathrm{FVC}$ for SNPs in seven previously unrecognized independent loci (GPR126, ADAM19, AGER-PPT2, FAM13A, PTCH1, PID1 and HTR4) and with $\mathrm{FEV}_{1}$ for one previously unrecognized independent locus annotated by at least three genes (INTS12-GSTCD-NPNT). The SpiroMeta consortium independently reported genome-wide significant associations of GSTCD, HTR4, AGER, TNS1 and THSD4 with $\mathrm{FEV}_{1} / \mathrm{FVC}$ and $\mathrm{FEV}_{1}$ (companion paper in this issue, ref. 15). Both consortia confirm previous GWAS findings implicating the HHIP region for $\mathrm{FEV}_{1} / \mathrm{FVC}^{10}$.

Several SNPs near the gene encoding the hedgehog interacting protein $(H H I P)$ were associated with $\mathrm{FEV}_{1} / \mathrm{FVC}$ at genome-wide significance in CHARGE and SpiroMeta, confirming earlier GWAS findings in $\mathrm{FHS}^{10}$. The hedgehog $(\mathrm{Hh})$ signaling pathway is crucial in several embryonic development processes, including the branching morphogenesis of the lung ${ }^{21,22}$. Furthermore, several polymorphisms in three genes in the Hh signaling pathway ( $I H H, H H I P$ and $P T C H 1)$ were significantly associated with adult height in a previous GWAS $^{23}$. Several PTCH1 SNPs were also significantly associated with $\mathrm{FEV}_{1} / \mathrm{FVC}$ in CHARGE, but these associations were not confirmed in SpiroMeta ${ }^{15}$. Epithelial cells produce $\mathrm{Hh}$ protein, which binds to its membrane receptor (encoded by PTCH1) on mesenchymal cells and orchestrates tissue and organ patterning. Hh pathway dysfunction during the fetal stage in humans is responsible for severe lung malformations ${ }^{24,25}$. In adults, the $\mathrm{Hh}$-signaling pathway may participate in the response of the airway epithelium to injury, such as smoking and hyperoxia ${ }^{26,27}$.

A nonsynonymous AGER SNP (rs2070600) was associated with $\mathrm{FEV}_{1} / \mathrm{FVC}$ at genome-wide significance in our study and independently confirmed in SpiroMeta ${ }^{15}$. The AGER protein, a membranebound or soluble pattern-recognition receptor, belongs to the immunoglobulin superfamily of cell surface receptors. The SNP rs2070600 has functional significance: for example, it promotes higher ligand affinity and the production of proinflammatory proteins upon activation ${ }^{28}$. In healthy adult mice and humans, AGER is highly expressed in the lungs ${ }^{29}$, and its absence contributes to the pathogenesis of idiopathic pulmonary fibrosis ${ }^{30,31}$. AGER signaling is involved in host defense, inflammation and tissue remodeling, processes that are relevant to accelerated decline in pulmonary function with age.

Polymorphisms in HTR4 were associated with $\mathrm{FEV}_{1} / \mathrm{FVC}$ at genome-wide significance in the joint meta-analysis of CHARGE and SpiroMeta results. HTR4 encodes a G-coupled transmembrane receptor that regulates cyclic AMP production in response to 5-hydroxytryptamine (serotonin). Elevated levels of free serotonin have been found in the plasma of individuals with symptomatic asthma ${ }^{32}$, and serotonin signaling pathways involving HTR4 have been implicated in cholinergic and immune-mediated airway reactivity ${ }^{33,34}$. Upon activation by serotonin, HTR4 in human airway epithelial cells regulates the release of a proinflammatory cytokine, a signature characteristic of asthma ${ }^{35}$.

ADAM19 SNPs were associated with $\mathrm{FEV}_{1} / \mathrm{FVC}$ at genomewide significance in CHARGE and in the joint meta-analysis with SpiroMeta. ADAM19 is a member of 'a disintegrin and metalloprotease' (ADAM) family of membrane-anchored glycoproteins that control cell-matrix interactions and help regulate growth and 
morphogenesis. Polymorphisms in a gene that encodes for another ADAM family member, ADAM33, have been associated with bronchial hyper-responsiveness and accelerated lung function decline in those with asthma and in the general population ${ }^{36-38}$. ADAM19 has not been previously implicated in human pulmonary disorders, but it is abundantly expressed in alveolar epithelial cells and bronchial smooth muscle tissue ${ }^{39}$.

GPR126 polymorphisms were associated with $\mathrm{FEV}_{1} / \mathrm{FVC}$ at genomewide significance in CHARGE and in the joint meta-analysis with SpiroMeta. GPR126 belongs to a superfamily of G protein-coupled receptors involved in cell adhesion and signaling ${ }^{40}$. Although the precise function of this gene has not been elucidated, its expression in mice is temporally increased during embryonic organ development and is highest in the adult lungs ${ }^{41}$. In humans, recent GWAS have linked GPR126 variants with adult height, and more specifically with trunk height ${ }^{42-44}$. We adjusted all analyses for standing height. Therefore, we repeated analyses after adjusting for sitting height (a more reliable indicator of trunk height) for GPR126 SNPs in ARIC, where both height variables were measured, and associations with $\mathrm{FEV}_{1} / \mathrm{FVC}$ remained significant (data not shown). Thus, these associations are not likely to be due to residual confounding by trunk height.

Genome-wide significant associations with $\mathrm{FEV}_{1}$ were observed in CHARGE for numerous SNPs spanning at least three genes on chromosome $4 \mathrm{q} 24$, and these associations were significant for all eight SNPs tested for replication in SpiroMeta (Table 3). There is moderate to strong linkage disequilibrium among the chromosome 4q24 SNPs, and the specific genes influencing $\mathrm{FEV}_{1}$ remain speculative. The genes are ordered INTS12GSTCD-NPNT along chromosome 4q24, and joint meta-analysis with SpiroMeta showed that SNPs from the genes INTS12 and GSTCD had the most significant associations with $\mathrm{FEV}_{1}$ (Table 3 ). The product of INTS12 is a subunit of the integrator complex that associates with the C-terminal domain of RNA polymerase II and mediates 3 '-end processing of small nuclear $\mathrm{RNAs}^{45}$. The glutathione S-transferase C-terminal domain (GSTCD) could influence lung function via mechanisms involving the detoxification by glutathione $\mathrm{S}$-transferases of xenobiotics that might damage the lungs.

The most distal gene in the chromosome $4 \mathrm{q} 24$ region, NPNT, encodes nephronectin, which is expressed in fetal and adult lungs ${ }^{46,47}$. The NPNT SNP rs10516529 is located in a binding site for the transcription factor POU6F1 (also known as mPOU homeobox protein), which is known to be expressed in adult lungs and hypothesized to play a role in lung development ${ }^{48-50}$. A fourth predicted gene in the region, FLJ20184, is located proximal to the other three genes. Although FLJ20184 encodes a hypothetical protein of unknown function, FLJ20184 contains allelic variants associated with successful smoking cessation in a GWAS of patients in smoking cessation trials ${ }^{51}$.

The genetic factors identified here gave estimated effect sizes consistent with those for well-established risk factors for pulmonary function decline. Carrying one copy of an implicated reference allele resulted in a $\mathrm{FEV}_{1}$ difference ranging from $50-70 \mathrm{~mL}$. These effect sizes correspond to approximately 2.8-3.9 years of age-related decline in pulmonary function based on a mean decline of about $18 \mathrm{~mL} /$ year and to approximately 1.7-2.3 years of active smoking-related decline based on a mean decline of about $30 \mathrm{~mL} /$ year $^{52}$. Second-hand smoke exposure has also been associated with decline in $\mathrm{FEV}_{1}(15 \mathrm{~mL}$ decline for a 10 -year exposure in the home and $41 \mathrm{~mL}$ decline for a 10 -year workplace exposure $)^{53}$. For $\mathrm{FEV}_{1} / \mathrm{FVC}$, carrying one copy of an implicated reference allele resulted in a difference ranging from $0.30 \%$ to $1 \%$. The lower effect-size estimates are comparable with the mean $\mathrm{FEV}_{1} / \mathrm{FVC}$ decline related to second-hand smoking ( 0.35 for a 10 -year exposure in the home and 0.14 for a 10 -year workplace exposure $)^{53}$.
These comparisons demonstrate that the identified genetic factors have a moderate impact on pulmonary function. Individuals carrying these polymorphisms will have lower pulmonary function than predicted at a given age, thus placing them at greater risk for developing COPD and at a greater risk of mortality ${ }^{2,3}$.

A GWAS of COPD identified CHRNA3-CHRNA5 on chromosome 15 as a susceptibility locus ${ }^{11}$. CHRNA3-CHRNA5 has also been associated with nicotine dependence ${ }^{12,13}$. In CHARGE, one identified SNP in this locus ( $r$ 1051730) was associated with $\mathrm{FEV}_{1} / \mathrm{FVC}(P=0.00070)$ and $\operatorname{FEV}_{1}(P=0.016)$, whereas the other identified SNP in this locus (rs8034191) was not associated with $\mathrm{FEV}_{1} / \mathrm{FVC}(P=0.11)$ or $\mathrm{FEV}_{1}$ $(P=0.36)$. The nominal evidence for replication may reflect differences in study design and a potential gene-environment interaction involving smoking.

Our study has several important strengths. The CHARGE cohorts are well phenotyped with pulmonary function measures passing stringent quality control criteria, thus minimizing measurement error. Our large sample size of 20,890 participants offers a powerful resource to examine associations of common SNPs with modest to large effects ${ }^{14}$. Testing our most significant results in the SpiroMeta consortium provided independent replication of these associations. However, even with the large sample sizes in these combined consortia, we likely have insufficient power to detect associations of polymorphisms with small effect sizes or low frequencies.

Population-based cohorts are subject to population stratification, and analytic steps were taken to minimize this potential bias. Cohortspecific $\lambda_{\text {gc }}$ values were low (1.00-1.05), and a genomic control adjustment was made in the meta-analyses to reduce inflation in the test statistics. The two largest cohorts, with the largest (albeit modest) $\lambda_{\mathrm{gc}}$ values (ARIC and FHS), incorporated principal components as potential confounders in their cohort-specific association tests. Although we cannot eliminate the possibility that some findings are subject to residual confounding by population stratification, the Q-Q plots showing deviations between observed and expected $P$ values for many high- to moderate-signal SNPs and the replication of association for multiple top loci in SpiroMeta suggest a multifactorial influence on pulmonary function.

Our study identified several previously unrecognized loci related to two clinically important pulmonary function measures with evidence for replication, including GPR126, ADAM19, AGER-PPT2 and HTR4 for $\mathrm{FEV}_{1} / \mathrm{FVC}$ and INTS12-GSTCD-NPNT for $\mathrm{FEV}_{1}$ and confirmed previous reports of association with $\mathrm{FEV}_{1} / \mathrm{FVC}$ in the HHIP region. These loci include genes with biologically plausible functions, and their identification here encourages future investigations to examine the mechanisms underlying their influence on pulmonary function. Fine mapping of these regions is needed to identify and characterize functional variants. Understanding the genetic determinants of pulmonary function is paramount in identifying the biological mechanisms that lead to its decline and in ultimately lessening the mortality burden associated with reduced pulmonary function.

\section{METHODS}

Methods and any associated references are available in the online version of the paper at http://www.nature.com/naturegenetics/.

Note: Supplementary information is available on the Nature Genetics website.

\section{ACKNOWLEDGMENTS}

This work was supported in part by the Intramural Research Program of the US National Institute of Environmental Health Sciences, National Institutes of Health (NIH), Department of Health and Human Services (Z01ES043012). The ARIC study is carried out as a collaborative study supported by US NIH National Heart, Lung, 
and Blood Institute contracts N01-HC-55015, N01-HC-55016, N01-HC-55018, N01-HC-55019, N01-HC-55020, N01-HC-55021, N01-HC-55022, R01HL087641, R01HL59367 and R01HL086694; National Human Genome Research Institute contract U01HG004402; and US NIH contract HHSN268200625226C. The authors thank the staff and participants of the ARIC study for their important contributions, along with G. Chiu, D. Howard and M. Quibrera for their analytic contributions.

The CHS research reported in this article was supported by contract numbers N01-HC-85079 through N01-HC-85086, N01-HC-35129, N01 HC-15103, N01 HC-55222, N01-HC-75150, N01-HC-45133, grant numbers U01 HL080295 and R01 HL087652 from the US NIH National Heart, Lung, and Blood Institute, with additional contribution from the US NIH National Institute of Neurological Disorders and Stroke. A full list of principal CHS investigators and institutions can be found at http://www.chs-nhlbi.org/pi.htm. DNA handling and genotyping was supported in part by US NIH National Center for Research Resources grant M01-RR00425 to the Cedars-Sinai General Clinical Research Center Genotyping core and National Institute of Diabetes and Digestive and Kidney Diseases grant DK063491 to the Southern California Diabetes Endocrinology Research Center.

Research was conducted in part using data and resources from the FHS of the National Heart, Lung, and Blood Institute of the US NIH and Boston University School of Medicine. The analyses reflect intellectual input and resource development from the FHS investigators participating in the SNP Health Association Resource (SHARe) project. This work was partially supported by the US NIH National Heart, Lung, and Blood Institute's FHS (Contract No. N01-HC-25195) and its contract with Affymetrix, Inc. for genotyping services (Contract No. N02-HL-6-4278). A portion of this research used the Linux Cluster for Genetic Analysis (LinGA-II), which is funded by the Robert Dawson Evans Endowment of the Department of Medicine at Boston University School of Medicine and Boston Medical Center. J.B.W. is supported by a Young Clinical Scientist Award from the Flight Attendant Medical Research Institute (FAMRI).

The Rotterdam Study was supported from grants from the Netherlands Organisation of Scientific Research (NOW) Investments (175.010.2005.011, 911-03-012); the Research Institute for Diseases in the Elderly (014-93-015; RIDE2); the Netherlands Genomics Initiative (NGI)/NWO (050-060-810); Erasmus Medical Center, Erasmus University, Rotterdam, The Netherlands; Organization for the Health Research and Development (ZonMw); the Research Institute for Diseases in the Elderly (RIDE); the Ministry of Education, Culture and Science; the Ministry for Health, Welfare and Sports; the European Commission (DG XII); and the Municipality of Rotterdam. The authors thank P. Arp, M. Jhamai, M. Moorhouse, M. Verkerk, and S. Bervoets for their help in creating the Rotterdam GWAS database; T. A. Knoch, L. V. de Zeeuw, A. Abuseiris and R. de Graaf as well as their institutions, the Erasmus Computing Grid, Rotterdam, The Netherlands, and the national German MediGRID and Services@MediGRID part of the German D-Grid (German Bundesministerium fur Forschung und Technology) (\#01 AK $803 \mathrm{~A}-\mathrm{H}$ and \# 01 IG $07015 \mathrm{G}$ ) for access to grid resources.

\section{AUTHOR CONTRIBUTIONS}

ARIC: D.B.H., L.R.L., N.F., M.B.S., D.J.C., N.M.P., A.C.M., K.E.N. and S.J.L. CHS: S.A.G., K.D.M., R.G.B., B.M.P., J.I.R., P.L.E., S.R.H. and T.L. FHS: J.B.W., T.-h.C. and G.T.O. RS: M.E., Y.M.T.A.v.D., G.G.B., C.M.v.D., A.G.U., A.H., F.R. and B.H.C.S. Study design: T.L., B.H.C.S., G.T.O. and S.J.L. Data analysis: D.B.H., M.E., J.B.W., L.R.L., K.D.M., N.F. and T.-h.C. Drafting of manuscript: D.B.H., M.E., J.B.W. and S.A.G. Critical revision of manuscript: D.B.H., M.E., J.B.W., S.A.G., L.R.L., K.D.M., N.F., Y.M.T.A.v.D., T.-h.C., R.G.B., M.B.S., D.J.C., G.G.B., B.M.P., C.M.v.D., J.I.R., A.G.U., A.H., N.M.P., F.R., A.C.M., P.L.E., K.E.N., S.R.H., T.L., B.H.C.S., G.T.O. and S.J.L.

Published online at http://www.nature.com/naturegenetics /.

Reprints and permissions information is available online at http://npg.nature.com/ reprintsandpermissions /.

1. Wilk, J.B. et al. Evidence for major genes influencing pulmonary function in the NHLBI family heart study. Genet. Epidemiol. 19, 81-94 (2000).

2. Hole, D.J. et al. Impaired lung function and mortality risk in men and women: findings from the Renfrew and Paisley prospective population study. Br. Med. J. 313, 711-716 (1996).

3. Schünemann, H.J., Dorn, J., Grant, B.J., Winkelstein, W. Jr. \& Trevisan, M. Pulmonary function is a long-term predictor of mortality in the general population: 29-year follow-up of the Buffalo Health Study. Chest 118, 656-664 (2000).

4. DeMeo, D.L. \& Silverman, E.K. Genetics of chronic obstructive pulmonary disease. Semin. Respir. Crit. Care Med. 24, 151-160 (2003).
5. Pauwels, R.A., Buist, A.S., Calverley, P.M., Jenkins, C.R. \& Hurd, S.S. Global strategy for the diagnosis, management, and prevention of chronic obstructive pulmonary disease. NHLBI/WHO Global Initiative for Chronic Obstructive Lung Disease (GOLD) Workshop summary. Am. J. Respir. Crit. Care Med. 163, 1256-1276 (2001).

6. Silverman, E.K. \& Sandhaus, R.A. Clinical practice. Alpha1-antitrypsin deficiency. N. Engl. J. Med. 360, 2749-2757 (2009).

7. Crapo, R.O. Pulmonary-function testing. N. Engl. J. Med. 331, 25-30 (1994).

8. Pellegrino, R. et al. Interpretative strategies for lung function tests. Eur. Respir. J. 26, 948-968 (2005).

9. Wilk, J.B., Walter, R.E., Laramie, J.M., Gottlieb, D.J. \& O'Connor, G.T. Framingham Heart Study genome-wide association: results for pulmonary function measures. BMC Med. Genet. 8 Suppl 1, S8 (2007).

10. Wilk, J.B. et al. A genome-wide association study of pulmonary function measures in the Framingham Heart Study. PLoS Genet. 5, e1000429 (2009).

11. Pillai, S.G. et al. A genome-wide association study in chronic obstructive pulmonary disease (COPD): identification of two major susceptibility loci. PLoS Genet. 5 e1000421 (2009).

12. Berrettini, W. et al. Alpha-5/alpha-3 nicotinic receptor subunit alleles increase risk for heavy smoking. Mol. Psychiatry 13, 368-373 (2008).

13. Saccone, S.F. et al. Cholinergic nicotinic receptor genes implicated in a nicotine dependence association study targeting 348 candidate genes with 3713 SNPs. Hum. Mol. Genet. 16, 36-49 (2007).

14. Psaty, B.M. et al. Cohorts for Heart and Aging Research in Genomic Epidemiology (CHARGE) Consortium: design of prospective meta-analyses of genome-wide association studies from 5 cohorts. Circ. Cardiovasc. Genet. 2, 73-80 (2009).

15. Repapi, E. et al. Genome-wide association study identifies five new loci associated with lung function. Nat. Genet. advance online publication, doi:10.1038/ng.501 (13 December 2009).

16. Kent, W.J. et al. The human genome browser at UCSC. Genome Res. 12, 996-1006 (2002).

17. Eyre, T.A. et al. The HUGO gene nomenclature database, 2006 updates. Nucleic Acids Res. 34, D319-D321 (2006).

18. Ramensky, V., Bork, P. \& Sunyaev, S. Human non-synonymous SNPs: server and survey. Nucleic Acids Res. 30, 3894-3900 (2002).

19. Ferguson, G.T., Enright, P.L., Buist, A.S. \& Higgins, M.W. Office spirometry for lung health assessment in adults: a consensus statement from the National Lung Health Education Program. Chest 117, 1146-1161 (2000).

20. Hankinson, J.L., Odencrantz, J.R. \& Fedan, K.B. Spirometric reference values from a sample of the general U.S. population. Am. J. Respir. Crit. Care Med. 159, 179-187 (1999).

21. Chen, M.H., Wilson, C.W. \& Chuang, P.T. SnapShot: hedgehog signaling pathway. Cell 130, 386 (2007).

22. Warburton, D. et al. Molecular mechanisms of early lung specification and branching morphogenesis. Pediatr. Res. 57, 26R-37R (2005).

23. Weedon, M.N. et al. Genome-wide association analysis identifies 20 loci that influence adult height. Nat. Genet. 40, 575-583 (2008).

24. Villavicencio, E.H., Walterhouse, D.O. \& lannaccone, P.M. The sonic hedgehogpatched-gli pathway in human development and disease. Am. J. Hum. Genet. 67 1047-1054 (2000)

25. Whitsett, J.A., Wert, S.E. \& Trapnell, B.C. Genetic disorders influencing lung formation and function at birth. Hum. Mol. Genet. 13(Spec. No. 2), R207-R215 (2004).

26. Lemjabbar-Alaoui, H. et al. Wnt and Hedgehog are critical mediators of cigarette smoke-induced lung cancer. PLoS One 1, e93 (2006).

27. Pogach, M.S., Cao, Y., Millien, G., Ramirez, M.I. \& Williams, M.C. Key developmental regulators change during hyperoxia-induced injury and recovery in adult mouse lung. J. Cell. Biochem. 100, 1415-1429 (2007).

28. Hofmann, M.A. et al. RAGE and arthritis: the G82S polymorphism amplifies the inflammatory response. Genes Immun. 3, 123-135 (2002).

29. Chavakis, T. et al. The pattern recognition receptor (RAGE) is a counterreceptor for leukocyte integrins: a novel pathway for inflammatory cell recruitment. J. Exp. Med. 198, 1507-1515 (2003).

30. Englert, J.M. et al. A role for the receptor for advanced glycation end products in idiopathic pulmonary fibrosis. Am. J. Pathol. 172, 583-591 (2008).

31. Queisser, M.A. et al. Loss of RAGE in pulmonary fibrosis: molecular relations to functional changes in pulmonary cell types. Am. J. Respir. Cell Mol. Biol. 39, 337-345 (2008).

32. Lechin, F., van der Dijs, B., Orozco, B., Lechin, M. \& Lechin, A.E. Increased levels of free serotonin in plasma of symptomatic asthmatic patients. Ann. Allergy Asthma Immunol. 77, 245-253 (1996).

33. Dupont, L.J. et al. The effects of $5-\mathrm{HT}$ on cholinergic contraction in human airways in vitro. Eur. Respir. J. 14, 642-649 (1999).

34. Idzko, M. et al. The serotoninergic receptors of human dendritic cells: identification and coupling to cytokine release. J. Immunol. 172, 6011-6019 (2004).

35. Bayer, H. et al. Serotoninergic receptors on human airway epithelial cells. Am. J. Respir. Cell Mol. Biol. 36, 85-93 (2007).

36. Van Eerdewegh, P. et al. Association of the ADAM33 gene with asthma and bronchial hyperresponsiveness. Nature 418, 426-430 (2002).

37. Jongepier, H. et al. Polymorphisms of the ADAM33 gene are associated with accelerated lung function decline in asthma. Clin. Exp. Allergy 34, 757-760 (2004).

38. van Diemen, C.C. et al. A disintegrin and metalloprotease 33 polymorphisms and lung function decline in the general population. Am. J. Respir. Crit. Care Med. 172, 329-333 (2005). 
39. Dijkstra, A. et al. Expression of ADAMs ("a disintegrin and metalloprotease") in the human lung. Virchows Arch. 454, 441-449 (2009).

40. Bjarnadottir, T.K., Fredriksson, R. \& Schioth, H.B. The adhesion GPCRs: a unique family of $G$ protein-coupled receptors with important roles in both central and peripheral tissues. Cell. Mol. Life Sci. 64, 2104-2119 (2007).

41. Moriguchi, T. et al. DREG, a developmentally regulated G protein-coupled receptor containing two conserved proteolytic cleavage sites. Genes Cells 9, 549-560 (2004).

42. Gudbjartsson, D.F. et al. Many sequence variants affecting diversity of adult human height. Nat. Genet. 40, 609-615 (2008).

43. Lettre, G. et al. Identification of ten loci associated with height highlights new biological pathways in human growth. Nat. Genet. 40, 584-591 (2008).

44. Soranzo, N. et al. Meta-analysis of genome-wide scans for human adult stature identifies novel Loci and associations with measures of skeletal frame size. PLoS Genet. 5, e1000445 (2009).

45. Baillat, D. et al. Integrator, a multiprotein mediator of small nuclear RNA processing, associates with the C-terminal repeat of RNA polymerase II. Cell 123, 265-276 (2005).
46. Brandenberger, R. et al. Identification and characterization of a novel extracellular matrix protein nephronectin that is associated with integrin alpha8beta1 in the embryonic kidney. J. Cell Biol. 154, 447-458 (2001).

47. Huang, J.T. \& Lee, V. Identification and characterization of a novel human nephronectin gene in silico. Int. J. Mol. Med. 15, 719-724 (2005).

48. Wey, E., Lyons, G.E. \& Schafer, B.W. A human POU domain gene, mPOU, is expressed in developing brain and specific adult tissues. Eur. J. Biochem. 220, 753-762 (1994).

49. Cardoso, W.V. Transcription factors and pattern formation in the developing lung. Am. J. Physiol. 269, L429-L442 (1995).

50. Warburton, D. et al. The molecular basis of lung morphogenesis. Mech. Dev. 92 55-81 (2000).

51. Uhl, G.R. et al. Genome-wide association for methamphetamine dependence: convergent results from 2 samples. Arch. Gen. Psychiatry 65, 345-355 (2008).

52. Kohansal, R. et al. The natural history of chronic airflow obstruction revisited: an analysis of the Framingham offspring cohort. Am. J. Respir. Crit. Care Med. 180, 3-10 (2009).

53. Eisner, M.D. et al. Secondhand smoke exposure, pulmonary function, and cardiovascular mortality. Ann. Epidemiol. 17, 364-373 (2007).

${ }^{1}$ Epidemiology Branch, National Institute of Environmental Health Sciences, National Institutes of Health, Department of Health and Human Services, Research Triangle Park, North Carolina, USA. ${ }^{2}$ Department of Epidemiology, Erasmus Medical Center, Rotterdam, The Netherlands. ${ }^{3}$ Departments of Neurology and Medicine, Boston University School of Medicine, Boston, Massachusetts, USA. ${ }^{4}$ Center for Lung Biology and ${ }^{5}$ Department of Medicine, University of Washington, Seattle, Washington, USA. ${ }^{6}$ Department of Epidemiology, University of North Carolina at Chapel Hill, Chapel Hill, North Carolina, USA. ${ }^{7}$ Cardiovascular Health Research Unit, University of Washington, Seattle, Washington, USA. ${ }^{8}$ Department of Respiratory Medicine, Ghent University and Ghent University Hospital, Ghent, Belgium. ${ }^{9}$ Pulmonary Center, Department of Medicine, Boston University School of Medicine, Boston, Massachusetts, USA. ${ }^{10}$ The National Heart, Lung, and Blood Institute's Framingham Heart Study, Framingham, Massachusetts, USA. ${ }^{11}$ Division of General Medicine and ${ }^{12}$ Division of Pulmonary, Allergy and Critical Care, Department of Medicine, College of Physicians and Surgeons, Columbia University, New York, New York, USA. 13Department of Epidemiology, Mailman School of Public Health, Columbia University, New York, New York, USA. ${ }^{14}$ Risk Assessment, Detection, and Intervention, H. Lee Moffitt Cancer Center and Research Institute, Tampa, Florida, USA. ${ }^{15}$ Department of Biostatistics, University of North Carolina at Chapel Hill, Chapel Hill, North Carolina, USA. ${ }^{16}$ Department of Epidemiology, University of Washington, Seattle, Washington, USA. ${ }^{17}$ Center for Health Studies, Group Health, Seattle, Washington, USA. ${ }^{18}$ Department of Health Services, University of Washington, Seattle, Washington, USA. ${ }^{19}$ Medical Genetics Institute, Cedars-Sinai Medical Center, Los Angeles, California, USA. ${ }^{20}$ Department of Internal Medicine, Erasmus Medical Center, Rotterdam, The Netherlands. ${ }^{21}$ Departments of Medicine and Epidemiology, Johns Hopkins University, Baltimore, Maryland, USA. ${ }^{22}$ Human Genetics Center, Division of Epidemiology and Disease Control, University of Texas Health Science Center at Houston, Houston, Texas, USA. ${ }^{23}$ College of Public Health, University of Arizona, Tucson, Arizona, USA. ${ }^{24}$ Carolina Center for Genome Sciences, University of North Carolina at Chapel Hill, Chapel Hill, North Carolina, USA. ${ }^{25}$ Department of Biostatistics, University of Washington, Seattle, Washington, USA. ${ }^{26}$ Netherlands Genomics Initiative-sponsored Netherlands Consortium for Healthy Aging, Rotterdam, The Netherlands. ${ }^{27}$ Inspectorate of Health Care, The Hague, The Netherlands. ${ }^{28}$ Laboratory of Respiratory Biology, National Institute of Environmental Health Sciences, National Institutes of Health, Department of Health and Human Services, Research Triangle Park, North Carolina, USA. ${ }^{29}$ These authors contributed equally to this work. ${ }^{30}$ These authors jointly directed this work. Correspondence should be addressed to S.J.L. (Iondon2@niehs.nih.gov). 


\section{ONLINE METHODS}

Pulmonary function measurements. Study design details of the participating CHARGE cohorts are described elsewhere ${ }^{14,54-59}$. Study protocols were approved by the relevant institutional review boards, and all participants provided written informed consent.

Pulmonary function testing was conducted by trained spirometry technicians at a single visit for RS and at more than one visit for ARIC, CHS and FHS. FEV $1 /$ FVC and FEV $_{1}$ measures meeting American Thoracic Society or European Respiratory Society criteria for acceptability were tested for association with SNPs in participants of European ancestry who were successfully genotyped and provided informed consent for genetic testing.

In ARIC and CHS, pulmonary function measures and questionnaire data from the baseline visit were analyzed. ARIC measurements were made with a Collins Survey II water-seal spirometer (Collins Medical, Inc.) and PulmoScreen II software (PDS Healthcare Products, Inc.) ${ }^{60}$. CHS measurements were made with a Collins Survey I water-seal spirometer (Collins Medical, Inc.) and software from S\&M Instruments ${ }^{61,62}$.

In three generations of families participating in the FHS, data from the most recent examination were analyzed. Eligible examinations providing spirometry and questionnaire data included examinations 13, 16, 17 and 19 in the original cohort (in approximate 2-year intervals); examinations 3, 5, 6 and 7 in the offspring generation (in approximate 4-year intervals); and the one examination completed to date for the third generation. Equipment used in the standard protocol evolved as technology improved over the decades of study ${ }^{63}$. A Collins Survey water-filled spirometer (Collins Medical, Inc.) was used for most examinations, with measurements made by Eagle II microprocessor (Collins Medical, Inc.) or by software from the S\&M Instruments. In more recent examinations, a Collins Comprehensive Pulmonary Laboratory dry rolling-seal spirometer and Collins 2000 Plus/SQL Software (Collins Medical, Inc.) were used.

In RS, pulmonary function was measured at the fourth center visit of participants from the original cohort (RS-I) and the second center visit of participants from the first extension cohort (RS-II). Spirometry was performed using a SpiroPro portable spirometer (Erich Jaeger GmbH) ${ }^{64,65}$.

Genotyping, imputation and quality control. Different genotyping platforms were used across the cohorts ${ }^{14}$ (Table 1). Imputation was conducted using either $\mathrm{MACH}^{66}$ or BIMBAM ${ }^{67}$ to generate approximately 2.5 million autosomal SNP genotype dosages for meta-analysis. The imputation methods perform similarly, although MACH generally produces higher accuracy rates than the imputation process used in BIMBAM (fastPHASE) ${ }^{68}$. Differing imputation methods across cohorts is not a source of bias for meta-analysis because all comparisons using the imputed data are within-cohort comparisons.

ARIC. Among 8,861 self-identified white ARIC participants genotyped, 8,127 participants remained in the study after exclusions for call rate $<95 \%$, genotypic and phenotypic sex mismatch, discordances with previous genotype data, suspected first-degree relative of an included individual based on genotype data, more than 8 s.d. for any of the first ten principal components using EIGENSTRAT ${ }^{69}$, or outlying average identity-by-state estimates using PLINK $^{70}$. Of these, 7,980 participants had available pulmonary function measures and complete covariate information.

A total of 704,588 autosomal genotyped SNPs remained after exclusions for call rate $<95 \%$, MAF $<1 \%$, Hardy-Weinberg equilibrium (HWE) $P<10^{-5}$ or lack of strand annotation. MACH (version 1.00.16) ${ }^{66}$ was used to impute all autosomal SNPs with reference to HapMap CEU (release 21, build 35) (1 $^{71}$ from these 704,588 SNPs. Imputed SNPs failing additional quality control criteria (monomorphism, HWE $P<10^{-6}$, or genotype frequencies between two genotyping phases differing by $P<10^{-6}$ ) were excluded, leaving 2,515,866 genotyped or imputed SNPs for analysis.

CHS. CHS genotyped 3,980 participants free of cardiovascular disease at baseline with available DNA and consent to genetic testing. After exclusions for call rate $<95 \%$, sex mismatch or discordance with prior genotyping, 3,291 self-identified white participants remained. Of these, 3,140 had pulmonary function measures and complete covariate information.

A set of 306,655 autosomal genotyped SNPs remained after exclusions for call rate $<97 \%$, HWE $P<10^{-5}$, more than two duplicate errors or mendelian inconsistency (for reference HapMap CEU trios) ${ }^{71}$, heterozygote frequency $>0$ or no mapping in dbSNP. Imputation of autosomal SNPs was based on these 306,655 SNPs using BIMBAM (version 0.99) ${ }^{67}$ with reference to HapMap CEU (release 22, build 36$)^{71}$. The analysis dataset included 2,543,887 genotyped or imputed SNPs.

FHS. A total of 8,481 participants remained after exclusions for call rate $<97 \%$, heterozygosity $>5$ s.d. from the mean, or excessive non-inheritance. The analysis dataset included 7,694 participants with complete spirometry and covariate data.

$\mathrm{MACH}$ (version 1.00.15) ${ }^{66}$ was used for imputation based on 378,163 autosomal SNPs remaining after exclusions for HWE $P<10^{-6}$, call rate $<97 \%$, differential missingness related to genotype (mishap procedure in PLINK ${ }^{70}$ ) with $P<10^{-9}$, mendelian errors $>100$, MAF $<1 \%$ or absence from HapMap. Two hundred unrelated individuals with high call rates were used to infer model parameters, which were subsequently applied to all 8,481 individuals. Imputation, using HapMap CEU (release 22, build 36) ${ }^{71}$, produced genotype dosages on 2,543,887 genotyped or imputed SNPs.

$R S$. All RS participants with available DNA were genotyped; 5,974 RS-I participants and 2,157 RS-II participants remained after exclusion for call rate $<97.5 \%$, excess autosomal heterozygosity, sex mismatch or outlying identity-by-state clustering estimates. Of these, 1,224 RS-I participants and 852 RS-II participants had pulmonary function measures and complete covariate information.

After exclusions for call rate $<98 \%$, HWE $P<10^{-6}$, and $\mathrm{MAF}<1 \%, 512,349$ autosomal SNPs in RS-I and 466,389 autosomal SNPs in RS-II were used for imputation in MACH (version 1.00.15 for RS-I and 1.00.16 for RS-II) ${ }^{66}$ with reference to the 2,543,887 SNPs of the HapMap CEU (release 22, build 36$)^{71}$.

Statistical analysis. In cross-sectional analyses, $\mathrm{FEV}_{1} / \mathrm{FVC}$ and $\mathrm{FEV}_{1}$ were tested for association with SNP genotypes using a 1-degree-of-freedom additive model of the dosage value (estimated reference allele count with a fractional value ranging from 0 to 2.0 ) as a predictor in linear regression models. Associations were examined overall and stratified into ever- and never-smokers. Overall models were adjusted for age, sex, standing height, smoking status (current, past or never-smoker) and pack-years of smoking. Current, past or never smoking was based on questionnaire responses, and pack-years were calculated for current and past smokers by multiplying smoking dose (packs per day) and duration (years smoked). Stratified models used the same covariates as the overall models, except that the ever-smoker stratum included adjustment for smoking status as current or past and the never-smoker stratum included no smoking-related covariates. Additional study-specific covariates included recruitment cohort (FHS), recruitment center (ARIC and CHS) and principal component eigenvalues for population stratification adjustments (ten components for ARIC and statistically significant components for FHS). Models were implemented using ProbABEL ${ }^{72}$ in ARIC, $\mathrm{R}^{73}$ in $\mathrm{CHS}$, linear mixed effects models with fixed effects for SNPs and random effects for individuals correlated within families ${ }^{74}$ in FHS, and MACH2QTL ${ }^{66}$ in RS as implemented in GRIMP ${ }^{75}$. In FHS, the kinship package in R generated a covariance matrix for each family based on the kinship coefficient for each relative pair. The kinship matrix, which includes the full set of family-specific covariance matrices, specified the covariance matrix for the random effects.

GWAS results from the four cohorts were combined using inverse variance weighted meta-analysis in METAL (see URLs). Meta-analysis was performed on approximately 2,534,500 SNPs after applying genomic control for each study and filtering out SNPs with extremely low imputation quality ratios $(<0.01)$ and MAFs $(<1 \%)$.

The genome-wide significance threshold was defined a priori as $P<5 \times 10^{-8}$, the Bonferroni adjustment for 1 million independent tests ${ }^{76}$. Information on SNP function and position relative to genes, microRNA and transcription factor binding sites was obtained using a Perl script (J.B.W.) that queries tables of the UCSC genome browser ${ }^{16}$ (hg18, March 2006 genome build, see URLs). Functional effects of nonsynonymous SNPs on protein structure and function were predicted using PolyPhen (see URLs) ${ }^{18}$.

Replication in the SpiroMeta consortium. We exchanged 30 SNPs for replication testing with the SpiroMeta consortium (companion manuscript ${ }^{15}$ ). No additional genotyping was required, as these SNPs were available from 
the SpiroMeta GWAS. We aimed to select two SNPs from each of the top genes implicated for $\mathrm{FEV}_{1} / \mathrm{FVC}$ or $\mathrm{FEV}_{1}$, with nearly all the genes exceeding genome-wide significance. The SNP with the lowest $P$ value in or near each gene was selected. A second SNP, genotyped (instead of imputed) in at least one cohort, was selected with preference for nonsynonymous SNPs and SNPs not in strong linkage disequilibrium with the first selected SNP. Only one SNP was available for AGER, PPT2, TSPYL4 and NT5DC1. Four SNPs were selected from two linkage disequilibrium blocks for the largest gene, GPR126. In total, 18 SNPs from 9 genes ( 8 independent loci) implicated for $\mathrm{FEV}_{1} / \mathrm{FVC}$ and 12 SNPs from 7 genes ( 3 independent loci) implicated for $\mathrm{FEV}_{1}$ were tested for replication.

Unlike CHARGE, SpiroMeta used normalized residuals as phenotypes, adjusted for age ${ }^{2}$ rather than age, and did not adjust for smoking. For better comparison, SpiroMeta conducted modified analyses following the CHARGE analytic method described above in 16,178 participants from adult cohorts with complete quantitative smoking data available. Results from the CHARGE GWAS and SpiroMeta replication were combined in a joint meta-analysis using inverse variance weighting with METAL. SpiroMeta results with $P<8.33 \times 10^{-4}$, based on an overly conservative Bonferroni correction for 60 tests (30 SNPs tested for association with two traits, $\mathrm{FEV}_{1} / \mathrm{FVC}$ and $\mathrm{FEV}_{1}$ ), or joint metaanalysis results with $P<5 \times 10^{-8}$ (genome-wide significance threshold), were considered statistically significant.

URLs. METAL, http://www.sph.umich.edu/csg/abecasis/metal/; UCSC Genome Browser, http://genome.ucsc.edu/; Polymorphism Phenotyping (PolyPhen), http://genetics.bwh.harvard.edu/pph/.

54. Anonymous. The Atherosclerosis Risk in Communities (ARIC) Study: design and objectives. The ARIC investigators. Am. J. Epidemiol. 129, 687-702 (1989).

55. Fried, L.P. et al. The Cardiovascular Health Study: design and rationale. Ann. Epidemiol. 1, 263-276 (1991).

56. Dawber, T.R. \& Kannel, W.B. The Framingham study. An epidemiological approach to coronary heart disease. Circulation 34, 553-555 (1966).

57. Feinleib, M., Kannel, W.B., Garrison, R.J., McNamara, P.M. \& Castelli, W.P. The Framingham Offspring Study. Design and preliminary data. Prev. Med. 4, 518-525 (1975).

58. Hofman, A. et al. The Rotterdam Study: objectives and design update. Eur. J. Epidemiol. 22, 819-829 (2007).
59. Hofman, A., Grobbee, D.E., de Jong, P.T. \& van den Ouweland, F.A. Determinants of disease and disability in the elderly: the Rotterdam Elderly Study. Eur. J. Epidemiol. 7, 403-422 (1991).

60. The National Heart and Blood Institute. Atherosclerosis Risk in Communities (ARIC) Study. Quality assurance and quality control, version 1.0. (ARIC Coordinating Center, School of Public Health, University of North Carolina, Chapel Hill, North Carolina, USA, 1989).

61. Enright, P.L., Kronmal, R.A., Higgins, M., Schenker, M. \& Haponik, E.F. Spirometry reference values for women and men 65 to 85 years of age. Cardiovascular health study. Am. Rev. Respir. Dis. 147, 125-133 (1993).

62. Enright, P.L., Kronmal, R.A., Higgins, M.W., Schenker, M.B. \& Haponik, E.F. Prevalence and correlates of respiratory symptoms and disease in the elderly. Cardiovascular Health Study. Chest 106, 827-834 (1994).

63. Givelber, R.J. et al. Segregation analysis of pulmonary function among families in the Framingham Study. Am. J. Respir. Crit. Care Med. 157, 1445-1451 (1998).

64. van Durme, Y.M. et al. Prevalence, incidence, and lifetime risk for the development of COPD in the elderly: the Rotterdam study. Chest 135, 368-377 (2009).

65. Miller, M.R. et al. Standardisation of spirometry. Eur. Respir. J. 26, 319-338 (2005).

66. Li, Y. \& Mach Abecasis, G.R. 1.0: rapid haplotype reconstruction and missing genotype inference. Am. J. Hum. Genet. S79, 2290 (2006).

67. Guan, Y. \& Stephens, M. Practical issues in imputation-based association mapping. PLoS Genet. 4, e1000279 (2008).

68. Pei, Y.F., Li, J., Zhang, L., Papasian, C.J. \& Deng, H.W. Analyses and comparison of accuracy of different genotype imputation methods. PLoS One 3, e3551 (2008).

69. Price, A.L. et al. Principal components analysis corrects for stratification in genomewide association studies. Nat. Genet. 38, 904-909 (2006).

70. Purcell, S. et al. PLINK: a tool set for whole-genome association and populationbased linkage analyses. Am. J. Hum. Genet. 81, 559-575 (2007).

71. The International HapMap Project. Nature 426, 789-796 (2003).

72. Aulchenko, Y.S., Ripke, S., Isaacs, A. \& van Duijn, C.M. GenABEL: an R library for genome-wide association analysis. Bioinformatics 23, 1294-1296 (2007).

73. Team, R.D.C. R: A language and environment for statistical computing. (R Foundation for Statistical Computing, Vienna, Austria, 2007).

74. Abecasis, G.R., Cardon, L.R., Cookson, W.O., Sham, P.C. \& Cherny, S.S. Association analysis in a variance components framework. Genet. Epidemiol. 21Suppl 1, S341-S346 (2001).

75. Estrada, K. et al. GRIMP: A web- and grid-based tool for high-speed analysis of large-scale genome-wide association using imputed data. Bioinformatics 15 2750-2752 (2009).

76. Pe'er, I., Yelensky, R., Altshuler, D. \& Daly, M.J. Estimation of the multiple testing burden for genomewide association studies of nearly all common variants. Genet. Epidemiol. 32, 381-385 (2008). 